\title{
COMMUNICATIONS
}

\section{OCULAR COMPLICATIONS OF DIABETES MELLITUS ${ }^{*}$}

$\mathrm{BY}$

\section{G. I. SCOTT}

Edinburgh

THERE is no doubt that the retinal changes which may occur in diabetic patients are by far the most important ocular complication of the disease.

It is now generally recognized that there is a type of retinopathy peculiar to diabetics, which is quite distinct from that occurring as a result of hypertension or arteriosclerosis.

Ballantyne and Loewenstein (1943), reviewing a large series of diabetic patients, found that 50 per cent. of those with retinal changes had normal blood pressure and that many showed no arteriosclerotic changes in the retinal vessels.

Wagener (1945), who at one time (Wagener and Wilde, 1921) had expressed the view that the retinopathy of diabetes was essentially that of arteriosclerosis, stated that, as a result of 25 years' further clinical experience, he was quite definitely of the opinion that neither arteriosclerosis nor hypertension produced retinopathy of the type characteristic of diabetes.

\section{Material of the Present Report}

Some years ago, in conjunction with Dr. Halliday Croom, I examined 124 diabetic patients, all of whom had had diabetes for periods varying from 15 to 26 years, and the findings in this group were recorded by one of us (Croom, 1950) in a consideration of the prognosis for the diabetic patient.

Since that time this group of patients under our observation has increased to 150 . In our study, the blood pressure was regarded as normal if the systolic pressure was below 140 and the diastolic below 90 . Patients were regarded as definitely hypertensive if the systolic pressure was 160 or more, or if the diastolic was 100 or more, and as doubtfully hypertensive if they exhibited blood pressures between these two levels.

* Received for publication August 26, 1953.

+ Opening paper read at the Annual Congress of the B.M.A., at Cardiff, in July, 1953 (Section of Ophthalmology). 
The distribution of the hypertensive cases is shown in Fig. 1; it will be noted that 35 of the 62 cases with retinopathy showed no evidence of hypertension.

In our first review of sixty of these patients (Croom and Scott, 1949), only믈 two showed albuminuria and oedema, which led us to the conclusion that

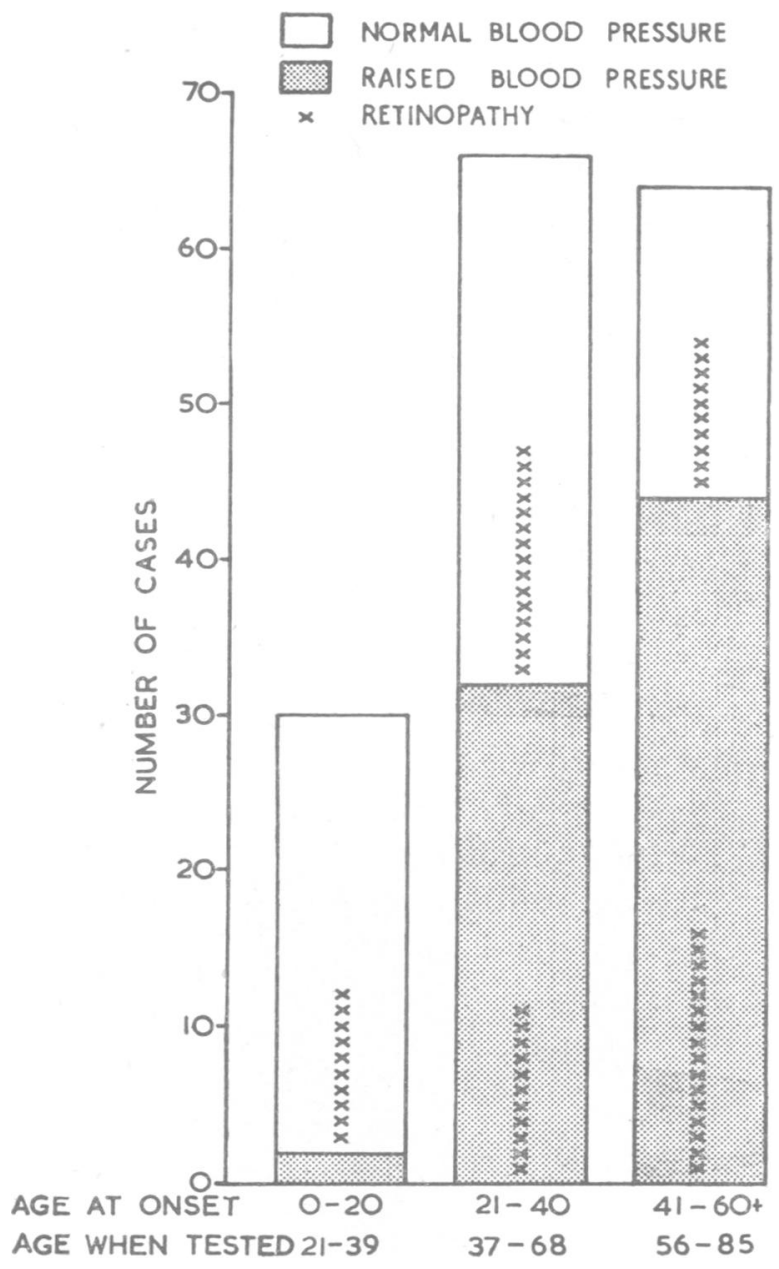

FIG. 1.-Distribution of cases with retinopathy, to illustrate that hypertension is not a necessary factor in the production of diabetic retinopathy. the nephrotic syndrome associa- $\frac{0}{0}$ ted with diabetes, while a pathological entity, was a relative $\frac{\bar{\omega}}{\bar{\phi}}$ clinical rarity, as had, in fact, been suggested by Kimmelstiel $\stackrel{\circ}{\circ}$ and Wilson (1936) in their original description of the con- $\vec{\omega}$ dition.

During the past few years, $\stackrel{\omega}{\omega}$ however, a few more of our $\vec{\sim}$ patients have developed this ơ complication. It would appear,o therefore, that the incidence of $\vec{\sigma}$ this syndrome is increasing, as, $\stackrel{\mathbb{\infty}}{\circ}$ in fact, one would expect in 3 view of the increased duration $\stackrel{\oplus}{\square}$ of life of the diabetic patient. $\frac{\delta}{0} \overrightarrow{0}$

We assessed the severity the diabetes by the dosage off insulin required, and the control⿳亠口冋口 of the condition by the general $\frac{\mathrm{O}}{\mathrm{O}}$ well-being of the patient, the $\stackrel{2}{\vec{P}}$ degree of glycosuria, and the 3 frequency of reactions.

\section{Ocular Pathology}

In this series of 150 patients, $\frac{\dot{2}}{3}$. as in our previous reviews, there was no significant correlation between the severity of the 9 ,disease and the development of $\frac{D}{2}$. cardiovascular or retinal complications. In so far as retinopathy is con- N cerned, I am sure that it occurs quite as frequently, if not more commonly, in patients with a mild degree of diabetes.

Wagener (1945) reviewed the ophthalmic findings in 1,021 diabetics examined at the Mayo Clinic during the year 1944. When the incidence of retino- $\frac{0}{0}$ pathy was correlated with the duration of the diabetes, it was found to be $\stackrel{\text { f }}{-}$ 10.7 per cent. in those who had had diabetes for less than one year, 22.0 per cent. in those who had had the disease for 1 to 10 years, and 73 per cent. in those who had been diabetics for more than 20 years. 
There thus seems to be no doubt that the incidence of retinopathy increases with the duration of the disease.

Within the space of two years (1943-1945), therefore, two factors were established: first, that a characteristic form of retinopathy can occur in diabetics which is independent of hypertension and unaccompanied by arteriosclerotic changes in the retinal vessels; secondly, that the incidence of this retinopathy varies with the duration of the disease.

We also know that the incidence of retinopathy is in no way related to the severity of the diabetes.

Joslin and others (1946) drew attention to the diabetic's increased expectation of life. In a review of 28,000 diabetics treated at Boston since 1898 , it was shown that immediately before the introduction of insulin in 1922 , a child who developed diabetes at the age of 10 years had an expectation of life of only 2.6 years, whereas by 1938 the expectation of life had increased to $39 \cdot 8$ years.

If one now considers these two factors, the increased expectation of life and the fact that the incidence of retinopathy increases with the duration of the disease, it is apparent why earlier observers maintained that retinopathy practically never occurred in young diabetics. The answer is simply that, before the introduction of insulin, the young diabetic rarely lived long enough to develop it.

Mackenzie (1877), in recording the clinical and histological findings in a patient who had died of diabetes mellitus, drew particular attention to the distension of the retinal veins and the presence of minute aneurysms on the capillaries. Nettleship (1877) suggested that these aneurysms might explain the occurrence of haemorrhages into the vitreous.

Mylius (1937) suggested that stasis in the veins was the essential factor in the causation of retinopathy in diabetes, but the significance of the venous changes was not truly appreciated until our thoughts were directed away from such factors as hypertension and retinal arteriosclerosis.

Ballantyne and Loewenstein (1943), recognizing that diabetic retinopathy and hypertensive retinopathy were two separate entities, rediscovered the capillary dilatations and demonstrated localized fatty degeneration in the walls of the small venules.

The more recent work of Ashton (1949) has confirmed that these lesions are in fact true micro-aneurysms and not merely capillary dilatations, and Ashton (1951) has shown that, while some of these lesions may commence as minute diverticulae arising on one side of the vessel wall, they arise more frequently from loops in the vessels which become varicose. Exudate then oozes through the vessel walls and the two loops become adherent, explaining how a large aneurysm may appear on an otherwise normal-looking capillary.

\section{Stages of Diabetic Retinopathy}

From the clinical point of view, I have already suggested (Scott, 1951) that 
we should adopt a classification (Fig. 2) slightly different from that used by the pathologist, to illustrate the various stages in the clinical course of this important complication of diabetes.

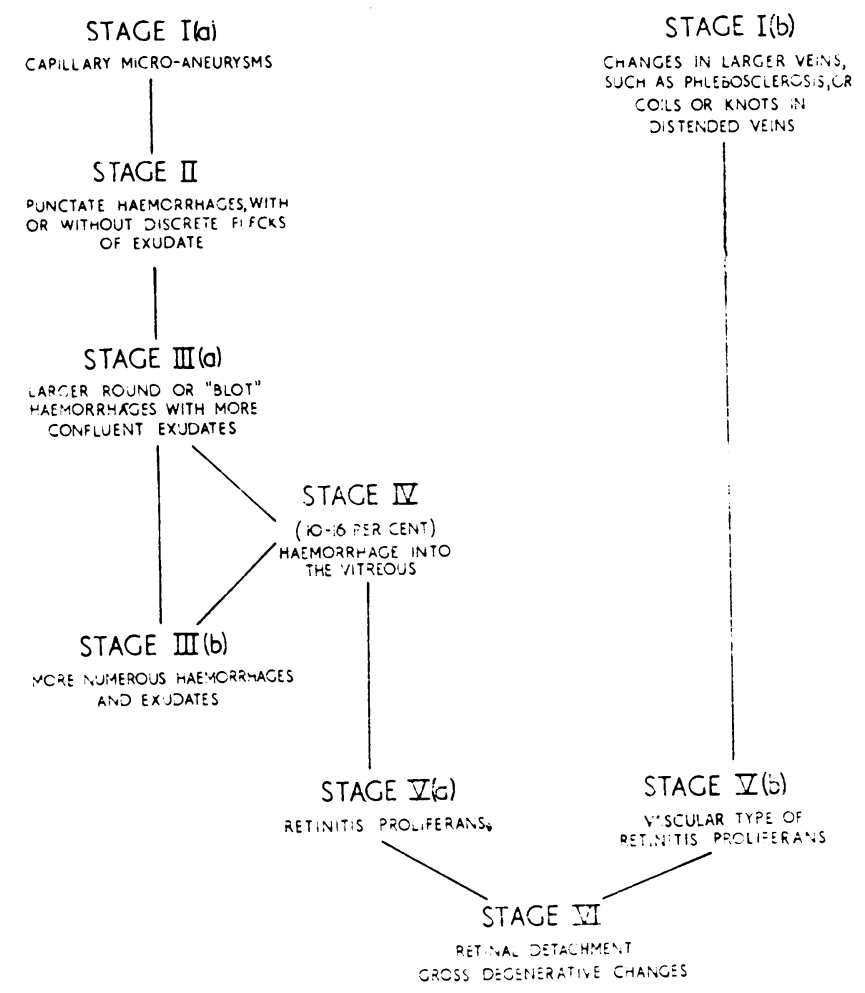

FIG. 2.-Clinical classification of the various stages of diabetic retinopathy.

At the commencement, the process is largely confined within the walls of the veins with the production of multiple micro-aneurysms, and perhaps a few haemorrhages (Stage I (a), see Fig. 3). In a smaller group of cases there may be characteristic, but not specific, changes in the larger veins, which may be distended and show loops or coils (Stage I $(b)$ ), as described by Ballantyne and Loewenstein (1943).

The next stage is the further escape of blood into the deeper layers of the retina, with or without the formation of exudates, the earliest stage consisting of punctate haemorrhages with perhaps a few flecks of exudate. (Stage II).

As this process progresses, the condition gradually changes to dot and blot haemorrhages, with more confluent exudates (Stage III (a), see Fig. 4).

In the minority, in which further deterioration occurs, the progress of the disease follows one of two very different courses. A certain number of cases show an exacerbation of the changes seen in Stage III $(a)$, the haemorrhages becoming more numerous and the exudates more confluent and more waxy in appearance, producing the text-book picture of the fundus appearances (Stage III $(b)$ ). 
FIG. 3.-Diabetic retinopathyStage I $(a)$.

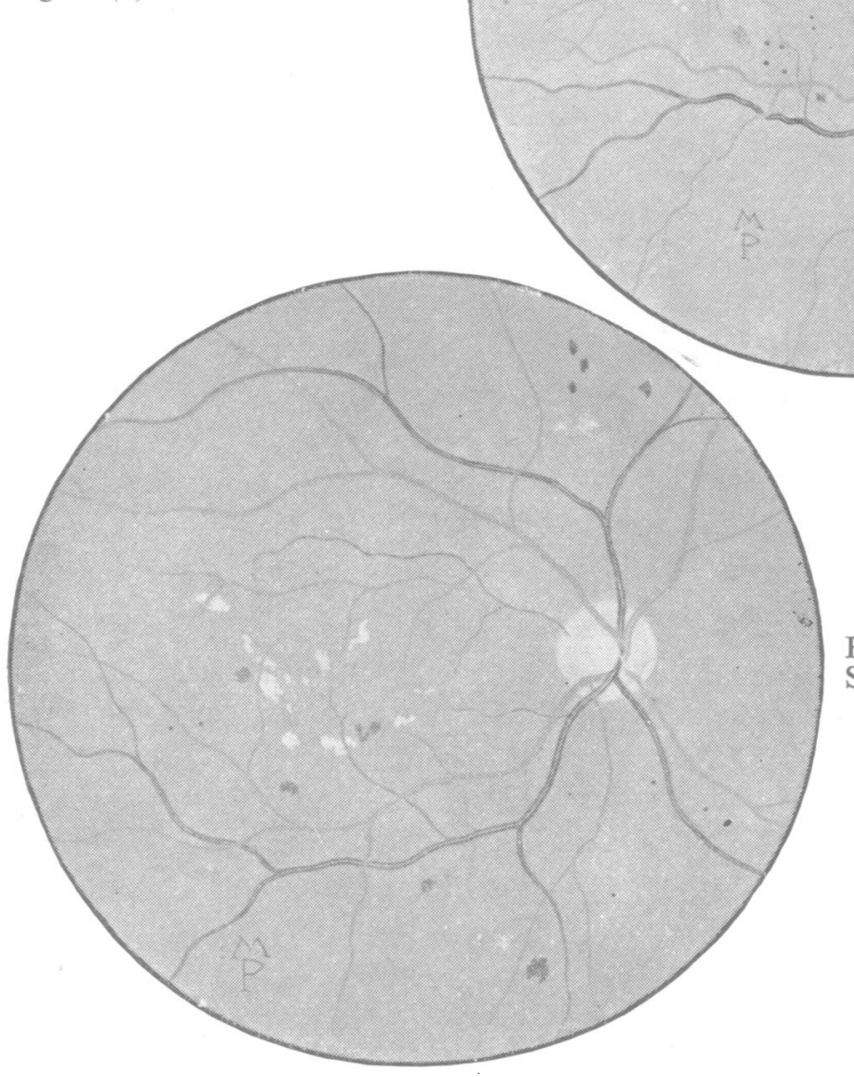

FIG. 4.-Diabetic retinopathyStage III $(a)$.

In other cases, however, a sudden haemorrhage into the vitreous may occur, which will inevitably be followed by the development of proliferative changes, resulting in that not so rare disaster, retinitis proliferans.

\section{Retinitis Proliferans}

It is, I think, important to recognize that bleeding into the vitreous may occur in the presence of relatively slight retinal changes such as I have described as occurring in Stage III (a), where the haemorrhages may be few in number. The only feature suggestive of possible disaster may be the fact that one or two of the haemorrhages are superficial and almost pre-retinal in position. As Hanum (1938) has expressed it: 
From these pre-retinal haemorrhages there seems to be but a floating transition to the apoplectiform haemorrhages which involve the vitreous and, probably always, give rise to proliferative changes.

Since the occurrence of this condition is dependent upon pre-existing retinal changes, its incidence primarily depends as in the case of the retinopathy, upon the duration of the diabetes.

Joslin and others (1952) review 326 cases of retinitis proliferans. In nearly 90 per cent. the patients had had diabetes for more than 5 years, and in 51 per cent. the duration of the diabetes exceeded 15 years. In nearly 40 per cent. this complication occurred before the 40th year, but in 71 cases $(22$ per cent.) it occurred after the age of 60 .

In our series, the ages of our six patients with retinitis proliferans were 27 , $33,42,46,57$, and 62 .

With regard to the likelihood of a diabetic patient with retinopathy developing retinitis proliferans, Wilson, Root, and Marble (1951) have reviewed a series of 247 patients with diabetes of 10-34 years' duration, among whom they found 158 (63.9 per cent.) with diabetic retinopathy. Of these 158 patients, 26 had developed retinitis proliferans, an incidence of 16.4 per cent. of the total number of cases with retinal changes.

In our Edinburgh series of 150 patients with long-standing diabetes, there were, as I have said, 62 cases $(41.3$ per cent.) with retinopathy, and six of these $(9 \cdot 7$ per cent.) developed retinitis proliferans.

\section{Prognosis as regards Vision}

It is important to remember, as Lawrence (1951) has emphasized, that the appearance of a few micro-aneurysms or haemorrhages in the fundi of a diabetic does not necessarily mean that he will suffer early impairment of vision, since serious macular involvement may not occur for many years.

Marked congestion of the veins and the occurrence of superficial blots of haemorrhage offer a much worse prognosis than scattered deep haemorrhages and exudates.

Even in the case of retinitis proliferans, useful vision may, in some cases, be preserved for as long as 5 or 6 years.

\section{Prevention and Treatment of Retinal Changes}

An increasingly high proportion of diabetics are showing such changes and, in the absence of adequate preventative measures or treatment, the number will undoubtedly increase.

In an analysis (Croom and Scott, 1949) of our first sixty cases of patients with long-standing diabetes, we found no correlation between the occurrence of vascular or retinal changes and the control of the diabetic state.

In our more recent review of 150 patients, we also found no significant difference in regard to "control ", in that, of the 62 cases with retinopathy, thirty were uncontrolled and 32 apparently well-controlled. 
We had, however, a considerable number of patients whom we had labelled "defaulters". While we knew that these patients were "well-controlled" or "not controlled" upon the occasions when they reported for review, they were irregular in their attendances for follow-up examination. We felt, therefore, that there was certainly a possibility that apparently "wellcontrolled" diabetics might not, in fact, be fully controlled during such periods of default. In fact, 51 per cent. of our patients had been "defaulters".

These patients were, as it happened, almost equally divided between our uncontrolled patients and those apparently controlled on the occasions upon which they did attend.

With regard to the incidence of degenerative changes, we found that 72 per cent. of the patients with retinopathy were defaulters, and that, of the 34 patients who were free from any sign of degenerative disease, only six were defaulters.

Although this group of cases is too small to enable one to draw definite conclusions, the findings in regard to "defaulters" certainly suggest that truly strict control of the diabetic state might lessen the incidence of degenerative changes. On the other hand, there is no doubt that in certain cases the disaster of haemorrhage into the vitreous occurs as a vascular accident in patients with only a few retinal haemorrhages, and may do so even in patients with perfectly controlled diabetes.

\section{Capillary Fragility}

There seems to be little doubt that capillary fragility is increased in a large proportion of patients with diabetic retinopathy. For example, Barnes (1950), in an analysis of 220 diabetics, found that, of eighty patients with retinal changes, 85 per cent. had increased capillary fragility. The average duration of the diabetes, in these cases, was $17 \cdot 2$ years. On the other hand, he found that oral administration of rutin, over long periods, had little effect in improving either capillary fragility or retinal changes.

In Edinburgh, we have found no evidence that rutin is of any practical value; though it might be worth while trying to control the increased capillary fragility in juvenile diabetics, before they have developed retinopathy, to see if such measures would prevent changes which one certainly cannot cure once they have become established.

\section{Changes in Refraction}

Horner (1873) drew attention to the sudden changes in refraction which might occur in diabetics. There is certainly no doubt that the alteration in refraction is due to changes in the lens. Elschnig (1923) reported a case in which the patient had previously had a cataract removed from one eye, and in whom these changes in refraction affected only the remaining lens.

As suggested by Duke-Elder (1925), it is probable that hydration of the lens by osmotic influences explains the mechanism of the change in most cases. 
With a rising blood-sugar, provided the water-balance of the body is reasonably maintained, the effective osmotic pressure of the tissue-fluids tends to fall owing to a loss of salts. The heavy loss of salt in the increased excretion of urine is not adequately replaced, although the bulk of fluid is made up by drinking water. The lowered osmotic pressure of the aqueous thus determines an inflow of water into the lens.

On the basis of the theory put forward by Duke-Elder, one can certainly explain the vast majority of cases in which transitory myopia develops with a rising blood-sugar, and in which the condition is reversed when the diabetes is treated. It is the sudden change to hypermetropia, soon after the commencement of treatment, which often worries the patient who suddenly becomes unable to read.

The points of practical importance are, firstly, to recognize that the sudden onset of myopia may be a sign of unsuspected diabetes, and secondly, to reassure the patient in whom hypermetropia suddenly develops when the disease is being treated. It is also important not to order any change of glasses for the latter disturbance, until 3 or 4 weeks have elapsed, during which time the refractive state of the eyes should have become stabilized.

While most cases can be explained on the theory expounded by Duke-Elder, there are those like that of Graham and Oakley (1938), in whom the reverse occurs, and the patient becomes less hypermetropic, or myopic, as the diabetic state is controlled. I have, myself, seen one or two similar cases, but these are exceptions to the general rule.

\section{Cataract in Diabetes}

True diabetic cataract, which is bilateral and often develops quickly, tends to occur in young patients with severe diabetes. This condition is relatively rare: the development of senile cataract is a problem of more practical importance though it seems no more common in diabetics than in non-diabetics in a similar age group. This view is supported by the findings of Waite and Beetham (1935), who found the incidence of senile cataract (2,000 diabetics) no greater than in non-diabetics of a comparable age.

On the other hand, my own impression is that senile cataract matures more quickly in the diabetic than in the non-diabetic patient.

Provided the diabetic with senile cataract has no other complications, such as retinopathy or change in the vessels of the iris, to which I shall refer later, the immediate result of operation is just as good, in my experience, in the diabetic as in the non-diabetic patient.

Bleeding from the iris is certainly more common in diabetics than in normal patients. On the other hand, my own experience is the same as that of De Voe (1942) who found that the presence of diabetes did not greatly alter the ultimate prognosis, although it might prolong the period of convalescence.

The most serious post-operative complication which may occur is that of iritis or iridocyclitis which may be severe and intractable. For this reason, 
it is, I think, of particular importance, before operating upon diabetic patients, to exclude, as far as possible, any focus of infection in, for example, the teeth, nasal sinuses, or renal tract.

Philps (1940) has recommended that, in diabetics, the iris should be interfered with as little as possible in order to minimize post-operative bleeding and I certainly agree with this recommendation where there is any sign of congestion or of new-vessel formation in the iris tissue. If, however, the iris appears healthy, then my own view is that it is more important to try to ensure that the lens is extracted without breaking the capsule, even if, in cases with a rigid pupil, it means doing a complete iridectomy. Just as the diabetic seems to be unduly susceptible to small foci of endogenous infection, severe post-operative iritis may be precipitated by remnants of lens material.

Apart from making a thorough search for possible foci of infection, the pre-operative care of the diabetic patient mainly devolves, as Kirby (1941) has emphasized, into the problem of getting the patient into the best possible state of general well-being.

This may involve a short period of observation before operation, with adjustment of the diet and insulin requirements to ensure that glycosuria is reduced to no more than a trace. On the morning of operation, the patient should be given a light carbohydrate breakfast with or without a small dose of soluble insulin. After operation, one should prescribe a light diet of approximately the same carbohydrate content as before operation, with enough insulin to avoid hypoglycaemia on the one hand, and marked hyperglycaemia on the other. As in the case of the non-diabetic, the diet should be such as to avoid unnecessary mastication.

\section{Other Ocular Complications}

The remaining ocular complications, are either rare, not amenable to treatment, or both.

Ocular Palsies.-Palsy of the external rectus is the commonest and recovery is the rule.

Amblyopia.-Although cases of chronic bilateral optic neuropathy have bzen described as occurring in diabetes, it is, as Walsh (1947) has suggested, extremely doubtful if there is such a thing as true diabetic optic neuritis. Certainly Waite and Beetham (1953) in their review of 2,000 diabetics did not encounter one case.

On the other hand, I have seen a number of cases of tobacco amblyopia develop during a phase of incomplete control of the diabetic state, and resolve completely, without cessation of smoking, as soon as control was regained. I have one patient, a heavy smoker, who develops such amblyopia periodically. If his period of poor control is prolonged, he cures his tobacco amblyopia by stopping smoking, but if he recovers control rapidly his amblyopia disappears without any alteration in his smoking habits.

As I have recently emphasized (Scott, 1953), bilateral toxic amblyopia 
in a diabetic patient should always arouse suspicion of the possibility of tobacco poisoning in a patient who smokes a pipe or cigars.

Rubeosis of the Iris.-This condition, in which there is a proliferation of new vessels in the iris, is rare but of serious significance, since it is always followed by glaucoma and is not amenable to operative treatment.

\section{Summary}

Retinopathy.-There is now no doubt that diabetic retinopathy is an entity quite distinct from that occurring as a result of hypertension. The findings in 150 diabetics, all of whom have had the disease for periods varying from 15 to 26 years, are discussed.

It seems to be generally agreed that incidence increases with the duration of the diabetes, and that it is in no way connected with the severity of the disease. The longer expectation of life for the diabetic since the introduction of insulin, means that retinopathy and other vascular changes now occur in the young as well as in the old, and have created a problem which can only be solved by intensive research.

The various stages in the development of the retinal changes are classified from a clinical point of view. In an appreciable number of cases, in the region of 10 to 16 per cent. of those with retinal changes, sudden haemorrhage into the vitreous may occur, and this will inevitably be followed by proliferative changes. It is emphasized, however, that, even in the presence of retinal changes, good visual acuity may be retained for many years.

The effect of " control " of the diabetic state, upon the incidence and course of the retinal changes, is discussed. The. view is expressed that this allimportant problem requires further investigation, but that it seems probable that the retinal and other degenerative vascular complications might be lessened by truly strict control of the diabetic state.

Transient Changes in Refraction.-The occurrence and clinical significance of such changes is described.

Cataract.-The effect of diabetes upon the incidence and development of senile lens opacities is discussed, as well as certain points in the preoperative care of the diabetic patient.

Other Complications.-The rarer conditions, such as toxic amblyopia, palsy, and rubeosis, are briefly considered.

\section{REFERENCES}

Ashton, N. (1949). British Journal of Ophthalmology, 33, 407.

(1951). Proc. roy. Soc. Med., 44, 747.

Ballantyne, A. J., and Loewenstein, A. (1943). Trans. ophthal. Soc. U.K., 63, 95.

BARNeS, R. H. (1950). Amer. J. med. Sci., 219, 368.

Croom, J. H. (1950). Edinb. Med. J., 57, 185.

- and ScOT, G. I. (1949). Lancet, 1, 555.

DeVoe, G. (1942). Arch. Ophthal., Chicago, 28, 1069.

DukE-ELDER, S. (1925). British Journal of Ophthalmology, 9, 167.

Elschnig, A. (1923). Med. Klin., 19, 17.

Graham, G., and OAKley, W. G. (1938). . St. Bart's Hosp. Rep., 71, 43.

Hanum, S. (1939). Acta ophthal., Kbh., Suppl. 16. 
Horner, J. F. (1873). Klin. Mbl. Augenheilk., 11, 488.

Joslin, E. P., Root, H. F., White, P., Marble, A., and Bailey, C. C. (1946). " Treatment of Diabetes Mellitus ", 8th ed. Kimpton, London. (1952). Ibid., 9th ed.

Kimmelstiel, P., and Wilson, C. (1936). Amer. J. Path., 12, 83.

KIRBY, D. B. (1941). Arch. Ophthal., Chicago, 25, 866.

LaWrence, R. D. (1951). Proc. roy. Soc. Med., 44, 742.

MACKenzIE, S. (1877). Roy. Lond. ophthal. Hosp. Rep., 9, 134.

Mylius, (1937). Klin. Mbl. Augenheilk., 98, 377.

NetTleship, E. (1877). Roy. Lond. Ophthal. Hosp. Rep., 9, 153.

PhILPS, A. S. (1940). British Journal of Ophthalmology, 24, 122.

Scotr, G. I. (1951). Proc. roy. Soc. Med., 44, 743. (1953). Practitioner, 170, 480.

WAGener, H. P. (1945). Proc. amer. Diabetes Ass., 5, 203. and Wilder, R. M. (1921). J. amer. med. Ass., 76, 515.

Waite, J. H., and Beetham, W. P. (1935). New Engl. J. Med., 212, 367.

WALSH, F. B. (1947). "Clinical Neuro-Ophthalmology", 1st ed. Williams and Wilkins, Baltimore.

Wilson, J. L., Root, H. F., and Marble, A. (1951). Amer. J. med. Sci., 221, 479. 